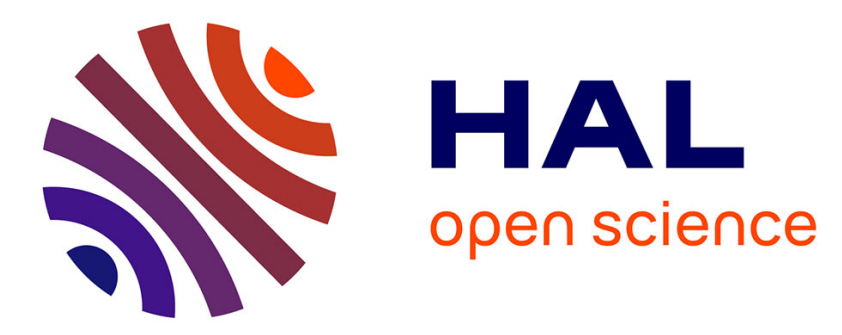

\title{
Phase space reconstruction of an experimental model of cardiac field potential in normal and arrhythmic conditions
}

\author{
Binbin Xu, Sabir Jacquir, Gabriel Laurent, Jean-Marie Bilbault, Stéphane \\ Binczak
}

\section{To cite this version:}

Binbin Xu, Sabir Jacquir, Gabriel Laurent, Jean-Marie Bilbault, Stéphane Binczak. Phase space reconstruction of an experimental model of cardiac field potential in normal and arrhythmic conditions. EMBC 2013 - 35th Annual International Conference of the IEEE Engineering in Medicine and Biology Society, Jul 2013, Osaka, Japan. pp.3274 - 3277, 10.1109/EMBC.2013.6610240 hal-00812198

\section{HAL Id: hal-00812198 \\ https://inria.hal.science/hal-00812198}

Submitted on 12 Apr 2013

HAL is a multi-disciplinary open access archive for the deposit and dissemination of scientific research documents, whether they are published or not. The documents may come from teaching and research institutions in France or abroad, or from public or private research centers.
L'archive ouverte pluridisciplinaire HAL, est destinée au dépôt et à la diffusion de documents scientifiques de niveau recherche, publiés ou non, émanant des établissements d'enseignement et de recherche français ou étrangers, des laboratoires publics ou privés. 


\title{
Phase space reconstruction of an experimental model of cardiac field potential in normal and arrhythmic conditions
}

\author{
Binbin $\mathrm{Xu}^{1}$, Sabir Jacquir ${ }^{2}$, Gabriel Laurent ${ }^{2}$, Jean-Marie Bilbault ${ }^{2}$, Stéphane Binczak ${ }^{2}$ *
}

\begin{abstract}
Cardiac arrhythmias are one of the most important death causes in the world. Compared to the numerical models, the experimental ones provide a more realistic tool to study the mechanisms of cardiac arrhythmias. The in vitro culture of cardiac cells developed on the Multi-Electrodes Array (MEA) constitutes a suitable model in this context. The extracellular field potential (EFP) acquired from the MEA can be used to measure the electrophysiological parameters of action potential. In this article, the stability of this experimental model is investigated using the phase space reconstruction in normal and in arrhythmia conditions. The results show that the embedding dimension of signal EFP changed slightly in both cases (normal conditions and arrhythmia). The parameter time lag $\tau$ in the normal conditions is lower than in the arrhythmia. The shape of attractors remains similar but disturbed in case of arrhythmia compared to the normal conditions.
\end{abstract}

\section{Introduction}

According to the recent report from the World Health Organization, the annual number of deaths due to cardiovascular disease will increase from 17 million in 2008 to 25 million in 2030 in the world. Among the death due to the cardiac problems, the arrhythmias play a major role, particularly in the atrial disorders. Facing to this challenge, many researches have been investigated in this field in order to understand the mechanism of these diseases. To achieve this goal, experimental models are much appreciated. They provide a more realistic situation than simulated ones. Ideally, it is better to conduct some in vivo studies in the heart. However, this is not always available and quite restricted by laws. Another serious problem of the in vivo studies is the heart beating. In order to overcome the limitations of the in vivo studies in the heart, the cardiac in vitro cultures are good alternatives and they permit even to study the arrhythmia at cellular level which could provide a better understanding of the mechanisms of arrhythmia generation and termination.

One of the technologies related to cardiac cultures is the MEA (Micro-Electrodes Array) which enables to monitor the

\footnotetext{
${ }^{* 1}$ Team GéoStat, INRIA Bordeaux Sud-Ouest, France

${ }^{\dagger 2}$ CNRS UMR 5158 Dijon France, LE2I Université de Bourgogne, Dijon France. Corresponding author, s jacquir@u-bourgogne. fr
}

culture for a longer time and is also less invasive compared to conventional electrophysiological methods as intracellular recording and patch-clamp. In a series of works $[1,2]$, we use our platform MEA to study the electrical impulse propagation (extracellular field potential, EFP) in cardiomyocytes culture under basal conditions. The results showed that in normal conditions, the electrical activities in the culture can be represented by the propagation of planar waves (action potential). In later studies [3,4], cardiac arrhythmias (spiral waves, SW) have been induced in the cardiomyocytes culture by electrical stimulations, in agreement with results in [5] which showed that a rapid stimulation could alter cardiac conduction and induce then arrhythmias. These results showed that this experimental model provide an excellent tool to study the cardiac arrhythmias itself and the effect of electrical stimulation on it. In order to study the behavior and the stability of the experimental model, non-linear methods are applied to the acquired signals. As the physiological signals, generally acquired as time series, are often marked by significant non-linearity that conventional analysis methods often fail to identify. It is more suitable to use non-linear methods. One of the popular nonlinear methods is phase space reconstruction, which is a valuable tool for the studies of this kind of signals. For a dynamical system, the phase space consists of a set of points (every point corresponds to a system state) which forms trajectories of the system. It shows information such as the existence of an attractor for selected parameter values. So this paper is aimed to determine the system dimension of this experimental model and to characterize it by reconstructing its phase space, in two cases: normal condition and under electrical stimulations.

The paper is organized as follows. The Section 2 presents the cardiomyocytes culture preparing and the electrical stimulation to the culture to obtain arrhythmic phenomenon. The system dimension determination and the reconstruction of phase space are discussed in Section 3.

\section{Experimental models and system}

\subsection{Extracellular field potentials}

The MEA consists of 60 electrodes aligned in a matrix form $8 \times 8$ with an inter-electrodes distance of $100 \mu \mathrm{m}$. The planar electrodes have a diameter of $30 \mu \mathrm{m}$. The cultures from cardiac muscular cells were developed directly on the 
MEA [6]. Every acquired signal is in fact a local extracellular field potential (EFP), since it comes from several cells around the electrode. They correspond to the combination the 1st and 2nd order derivative of the action potential. In basal conditions, the EFP signals are quite regular (Fig. 1).

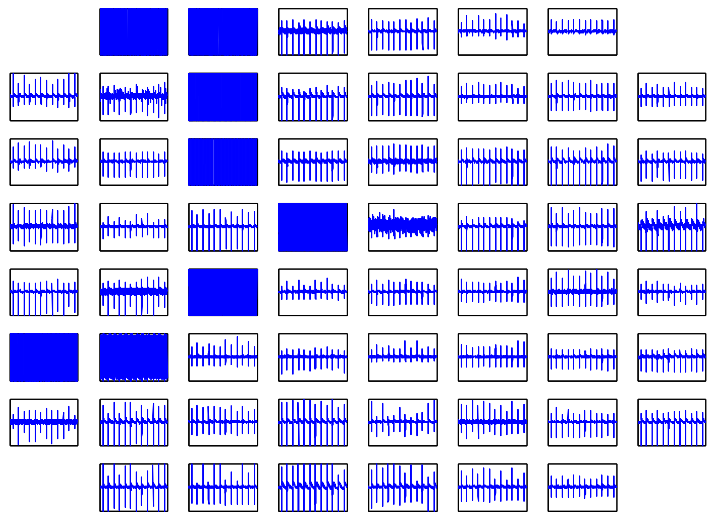

Figure 1: EFP in basal conditions. Every panel corresponding to an acquisition of $8 \mathrm{~s}$ from the real position of an electrode. (sampling frequency $500 \mathrm{~Hz}$ )

\subsection{Arrhythmic signals in cardiomyocytes culture induced by electrical stimulation}

To induce arrhythmia in heart or in cardiomyocytes culture, there exist generally two methods : by injection of specific drugs such as aconitine and acetylcholine etc. [7] and by vagal or electrical stimulation. We are interested in the method of electrical stimulation as rapid pacing with low energy can induce arrhythmia and promote it in isolated heart or in cardiac cultures $[3,4]$. In fact, these electrical stimulations can also be a potential method of atrial defibrillation. In recent years, the concept of sub-threshold stimulation with low amplitude and high frequency becomes more and more known and accepted [8-10]. In order to study experimentally the sub-threshold defibrillation, it is better to understand firstly the culture model itself under electrical stimulation. By electrical stimulation, cardiac arrhythmia can be induced in cardiac cultures (Fig. 2).

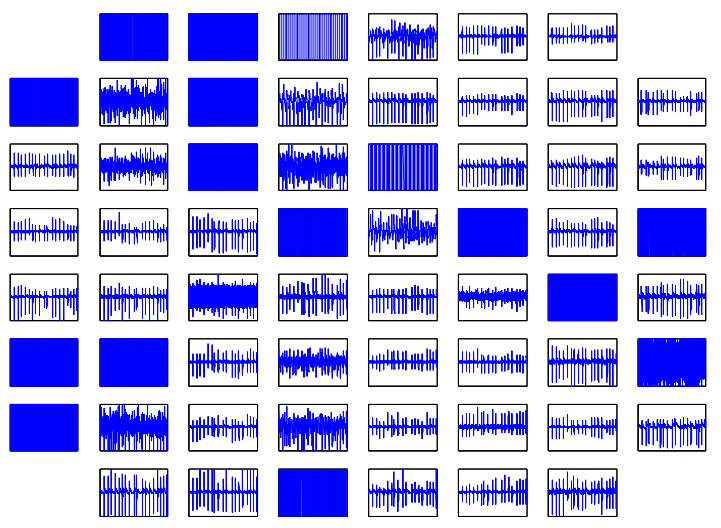

Figure 2: Arrhythmic signals after electrical stimulations.

\section{Phase space reconstruction}

The principle of phase space reconstruction is to transform the properties of a time series into topological properties of a geometrical object which is embedded in a space, wherein all possible states of the system are represented, each state corresponds to a unique point, and this reconstructed space sharing the same topological properties as the original space. Mathematically, the states of a $m$ dimensional dynamic system can only be characterized by $m$ independent quantities. Such a set of $m$ independent quantities represents the coordinates of the phase space (more exactly: the coordinates in a $m$ dimensional space).

One of the most used methods of phase space reconstruction is the time delay embedding. Since this method does not require that the treated system could be mathematically defined, explicitly, it fits well with 1D time series.

$$
\vec{X}(m, \tau)=[x(t), x(t+\tau), \ldots, x(t+\tau(m-1))], t=0,1,2, \ldots
$$

where $\vec{X}$ denotes the system states of time series $x(t)$, a function of its embedding dimension $m$ and time lag $\tau$. The reconstruction preserves the properties of the dynamic system that do not change under coordinate changes, that is to say, it keeps only the topological aspect instead of the geometric shape in phase space. To better present these signals in their phase space, the $m$ and $\tau$ should be determined very carefully. This is the purpose of the following paragraphs.

\subsection{Embedding dimension $m$}

As previously mentioned, a well-defined embedding dimension is essential to a phase space. For experimental data, we do not unfortunately know a priori the dimension of the studied system. The phase space reconstruction is based on the principle of connecting the state points to create attractor trajectories of the system, if $m$ is not large enough, the states points on the trajectories would be very close, there will be intersections between the trajectories of the attractor. A higher value of $m$, theoretically, is possible by just creating a larger space that contains minimal space. However, this will cause not only redundant problems, but also an efficiency issue, as it requires a much important and unnecessary computation power. The most common method used to estimate the embedding dimension is the method of False Nearest Neighbor (FNN) [11]. The criterion for choosing the value of $m$ corresponds to the minimum value of $m$ for which the FNN pass into is close to zero.

By the method on FNN, it is found that in normal condition and in case of arrhythmia, the embedding dimension of EFP depends on signals itself (Figures ??). The obtained values are all in the range of embedding dimension reported by others studies for cardiac signals [13]. Even $m$ changed slightly, it remains globally stable, which implies that the model is robust and stable. 

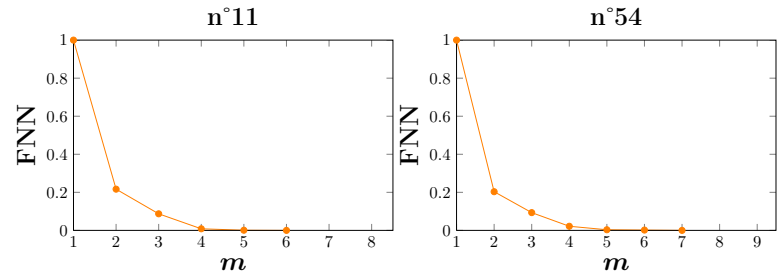

Figure 3: Estimation of $m$ by FNN, regular signals' examples, electrodes 11 and 54 .
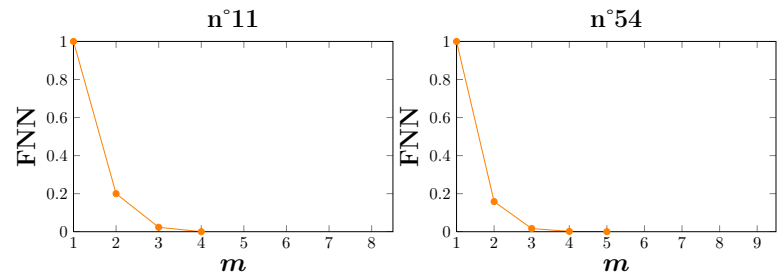

Figure 4: Estimation of $m$ by FNN, arrhythmic signals.

\subsection{Time lag $\tau$}

Non-linear variables change of a dynamic system will act as bases of coordinates. Ideally, this database should be independent of selected time lag $\tau$. The goal is to find the largest value of $\tau$ so that the resulting coordinates are relatively independent. If $\tau$ is too small, the trajectories of $x(t)$ and $x(t+\tau)$ close to one another, could even be indistinguishable and it will create redundant coordinates. For this reason, the basic criterion for choosing $\tau$ is that $x(t)$ and $x(t+\tau)$ should be independent. On the other hand, if $\tau$ is sufficiently too large, the two coordinates are statistically independent. This implies that the trajectories of attractor projected on the two axes are not correlated, which makes the reconstruction phase space useless.

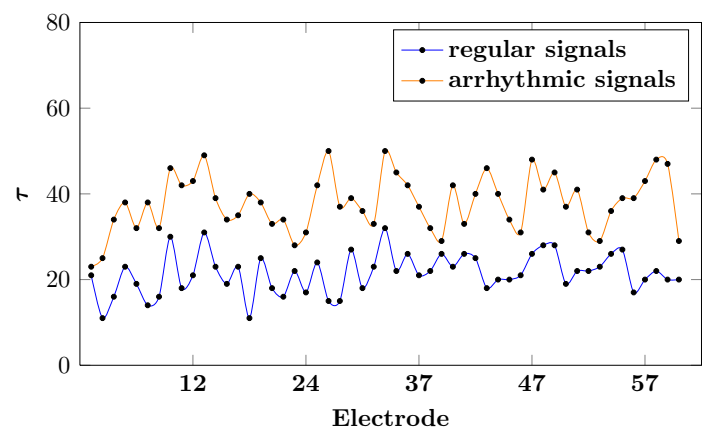

Figure 5: Comparison of $\tau$ by autocorrelation function (first $1 / e$ acrossing) for two type of EFP signals: regular signals and arrhythmic ones. $\tau$ is used as samples (sampling frequency $500 \mathrm{~Hz}$ ).

Without a priori knowledge of the system, especially for experimental data, it is not obvious to properly determine this parameter. The method by autocorrelation function is used here [14]. According to correlation theorem, it is known that under certain conditions two observations of the same process $\left(x\left(t_{1}\right), x\left(t_{2}\right)\right)$ or two functions $\left(x_{1}(t), x_{2}(t)\right)$ to describe this process may be identical, which means $x\left(t_{1}\right)=x\left(t_{2}\right)$ or $x_{1}(t)=x_{2}(t)$. Basically, the autocorrelation function $C(\tau)$ defines the similarity of a signal to itself but temporally shifted by $\tau$ which depends on the properties of treated data, it's necessary always to examine it by several methods in order to obtain a better estimation. Taking the signals that are previously studied, the estimation results of $\tau$ are presented in Fig. 5. By comparing these signals, we find that the values of $\tau$ for arrhythmic signals $(39 \pm 8)$ is higher than the ones for normal signals $22 \pm 6$. $\tau$ may serve as an indication of arrhythmia.

\subsection{Phase space reconstruction}

The reconstructed phase space are presented in Fig. 6 for regular signals and in Fig. 7 in case of cardiac arrhythmia.
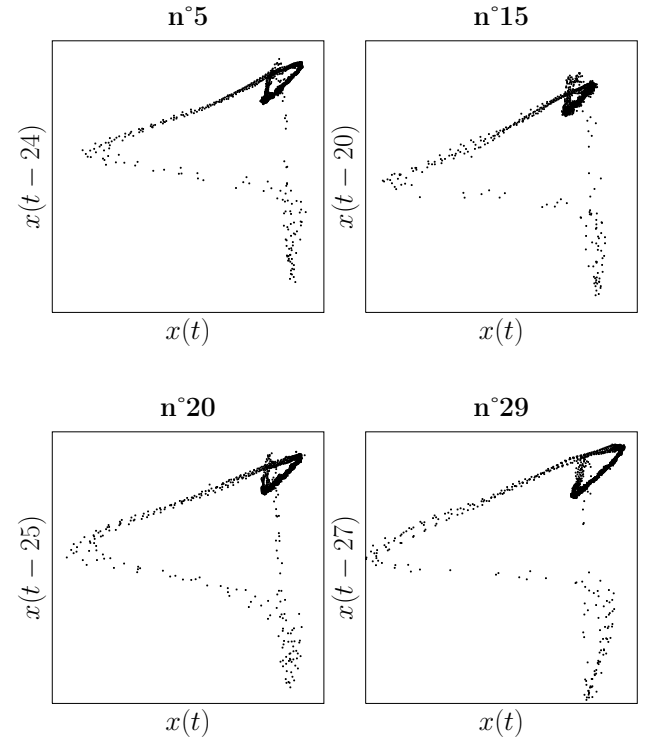

Figure 6: Phase space reconstruction for regular signals (from electrodes 5, 15, 20 and 29).

As shown in Fig. 6, the trajectories of the normal signals illustrate the existence of an attractor in their phase spaces. The global forms of these trajectories are rather triangular; the widths of the trajectories are relatively narrow, which means that the trajectories are stable. In case of arrhythmias (Fig. 7), the reconstructed phase space become very disturbed and are no longer regular. The differences are remarkable. However, the same attractors as the ones for regular signals are more or less found. Actually, if one compares the regular signals and arrhythmic signals, despite some variations in amplitude and periods, arrhythmic signals can be considered as "stable".

\section{Conclusion}

In our previous work [3], inducing cardiac arrhythmia by electrical stimulation is confirmed by our experimental studies. It showed by the resulting period-doubling route to chaos that in case of arrhythmia, EFP signals become chaotic. To reveal more information embedded in the time series, nonlin- 

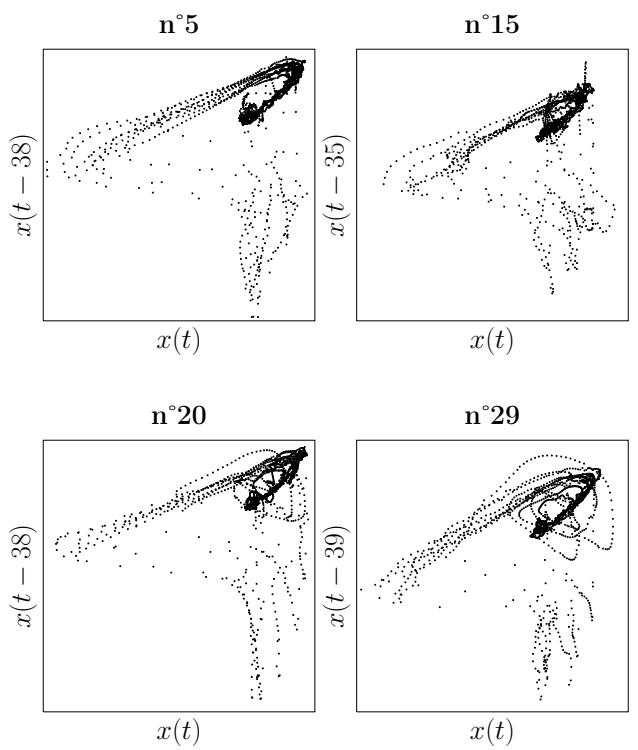

Figure 7: Phase space reconstruction for arrhythmic signals (from electrodes 5, 15, 20 and 29).

ear methods for physiological signals are more suitable than linear ones.

In case of arrhythmias induced by electrical stimulations, the embedding dimension of EFP signals changed slightly, but generally stable, which is a sign of the model stability. However, for the parameter "time lag" $\tau$, it is in opposite situation. For arrhythmic signals, the values of their $\tau$ are increased. So the change from normal situation to arrhythmia could be identified by $m$ and $\tau$, which would provide potential index for arrhythmia detection / identification.

The experimental model can be considered as "stable" : after the electrical stimulation, the global trajectories of EFP signals in the reconstructed phase space remain similar. In normal conditions, the signals are regular, their phase spaces have a triangular form, and the widths of the trajectories are relatively narrow. "Attractors" are formed at the upper part in the phase space. With the electrical stimulation, the trajectories of the model are very disturbed. They became much wider. However, their forms are more or less the same as in normal conditions; the global triangular form is kept, which reflected that the system dynamics of this model is generally stable.

In conclusion, the cardiomyocytes cultures are quite stable. The arrhythmic signals can be easily identified by time delay $\tau$. The electrical stimulation does not modify the global dynamics of the experimental model, which makes it be a robust tool for studying the mechanism of cardiac arrhythmia and the defibrillation by sub-threshold electrical stimulation.

\section{Acknowledgements}

B. Xu is financially supported by the French IHU in cardiac rhythmology L'Institut de rythmologie et modélisation cardiaque LIRYC and the regional council of Aquitaine.

\section{References}

[1] P. Athias, S. Jacquir, C. Tissier, D. Vandroux, S. Binczak, J. Bilbault, and M. Rossé, "Excitation spread in cardiac myocyte cultures using paired microelectrode and microelectrode array recordings," Journal of Molecular and Cellular Cardiology, vol. 42, no. 6, pp. S3-S3, 2007.

[2] S. Jacquir, C. Tissier, D. Vandroux, S. Binczak, J. Bilbault, M. Rossé, and P. Athias, "Paired microelectrodes and microelectrode array analysis of cardiac impulse propagation in cardiomyocyte cultures," Fundamental Clinical Pharmacology, vol. 22, no. 1, pp. 51-52, 2008.

[3] S. Jacquir, S. Binczak, B. Xu, G. Laurent, D. Vandroux, P. Athias, and J. Bilbault, "Investigation of micro spiral waves at cellular level using a microelectrode array technology," Int. J. Bifurcation Chaos, vol. 21, pp. 1-15, 2011.

[4] P. Athias, S. Jacquir, G. Laurent, D. Vandroux, S. Binczak, and J. Bilbault, "In vitro simulation of spiral waves in cardiomyocyte networks using multi-electrode array technology," European Journal of Heart Failure Supplements, vol. 8, p. $963,2009$.

[5] A. A. Kondratyev, J. G. C. Ponard, A. Munteanu, S. Rohr, and J. P. Kucera, "Dynamic changes of cardiac conduction during rapid pacing," American Journal of Physiology - Heart and Circulatory Physiology, vol. 292, no. 4, pp. H1796-H1811, 2007.

[6] P. Athias, D. Vandroux, C. Tissier, and L. Rochette, "Development of cardiac physiopathological models from cultured cardiomyocytes," Annales de Cardiologie et d'Angéiologie, vol. 55, pp. 90-99, apr 2006.

[7] M. Allessie, W. Lammers, F. Bonke, and J. Hollen, "Experimental evaluation of moe's multiple wavelet hypothesis of atrial fibrillation," Cardiac Electrophysiology and Arrhythmias. New York: Grune \& Stratton, pp. 265-276, 1985.

[8] G. Osipov, B. Shulgin, and J. Collins, "Controlled movement and suppression of spiral waves in excitable media," Phys. Rev. E, vol. 58, no. 6, pp. 6955-6958, 1998.

[9] T. K. Shajahan, A. R. Nayak, and R. Pandit, "Spiral-wave turbulence and its control in the presence of inhomogeneities in four mathematical models of cardiac tissue," PLOS ONE, vol. 4, p. 4738, mar 2009.

[10] B. Xu, S. Jacquir, G. Laurent, J.-M. Bilbault, and S. Binczak, "A hybrid stimulation strategy for suppression of spiral waves in cardiac tissue," Chaos, Solitons \& Fractals, vol. 44, pp. 633-639, aug 2011.

[11] M. B. Kennel, R. Brown, and H. D. I. Abarbanel, "Determining embedding dimension for phase-space reconstruction using a geometrical construction," Phys. Rev. A, vol. 45, pp. 3403-3411, mar 1992.

[12] O. Fojt and J. Holcik, "Applying nonlinear dynamics to ecg signal processing," Engineering in Medicine and Biology Magazine, IEEE, vol. 17, pp. 96 -101, march-april 1998.

[13] J. Fell, K. Mann, J. Röschke, and M. S. Gopinathan, Nonlinear analysis of continuous ECG during sleep I. Reconstruction, vol. 82, pp. 477-483. Springer-Verlag, 2000.

[14] A. M. Albano, J. Muench, C. Schwartz, A. I. Mees, and P. E. Rapp, "Singular-value decomposition and the grassbergerprocaccia algorithm," Phys. Rev. A, vol. 38, pp. 3017-3026, sep 1988. 